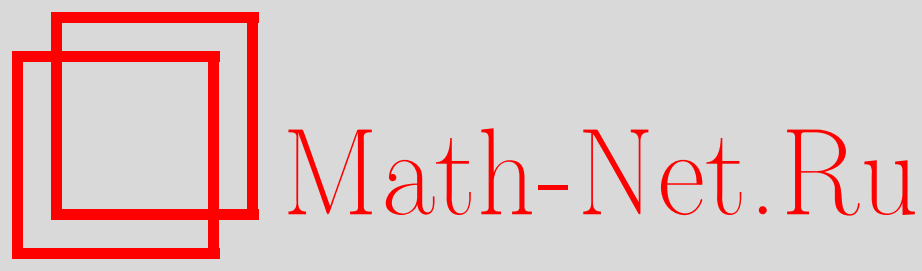

И. Я. Арефьева, И. В. Волович, О. В. Иноземцев, Эволюция голографических энтропийных величин для составных квантовых систем, ТМФ, 2018, том 197, номер 3, 510-517

DOI: https://doi.org/10.4213/tmf9613

Использование Общероссийского математического портала Math-Net.Ru подразумевает, что вы прочитали и согласны с пользовательским соглашением http://www . mathnet.ru/rus/agreement

Параметры загрузки:

IP : 3.80 .181 .102

26 апреля 2023 г., 15:00:27

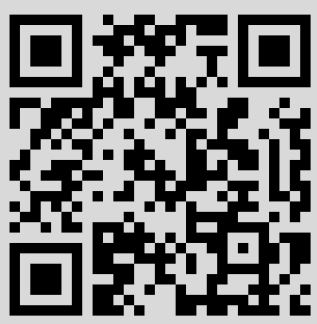




\title{
ЭВОЛЮЦИЯ ГОЛОГРАФИЧЕСКИХ ЭНТРОПИЙНЫХ ВЕЛИЧИН ДЛЯ СОСТАВНЫХ КВАНТОВЫХ СИСТЕМ
}

\begin{abstract}
Для системы, состоящей их трех частей, рассматриваются величины, основанные на энтропии зацепленности: трехчастичная информация, полная корреляция и так называемая защищенная монотонность. Для вычисления временной эволюции энтропии зацепленности при неравновесном нагреве используется голографический подход, что приводит к голографическим определениям перечисленных выше величин. Изучена их временная зависимость.
\end{abstract}

Ключевые слова: пространство Вадьи-АдС, трехчастичная информация, полная корреляция, защищенная монотонность.

DOI: https://doi.org/10.4213/tmf9613

\section{1. ВВЕДЕНИЕ}

Ранее в работе [1] мы рассматривали составную голографическую квантовую систему, одна часть которой состоит из одного отрезка, а другая - из двух отрезков. Для такой системы из трех отрезков мы исследовали поведение голографической взаимной информации между двумя частями системы. При этом, очевидно, три отрезка, составляющие систему, были неравноправны. Были обнаружены пять принципиально разных типов поведения для временной зависимости голографической взаимной информации.

В настоящей работе для системы, состоящей из трех отрезков, мы рассматриваем величины, являющиеся обобщениями взаимной информации, в которые все три отрезка входят одинаковым образом. Эти величины - трехчастичная информация, полная корреляция и защищенная монотонность (secrecy monotone). Интересно, что для полной корреляции и защищенной монотонности обнаружены в точности те же пять типов поведения, что и для голографической взаимной информации в [1]. Для трехчастичной информации удалось найти только четыре из пяти типов поведения.

Исследование выполнено за счет гранта Российского научного фонда (проект № 14-50-00005).

* Математический институт им. В. А. Стеклова Российской академии наук, Москва, Россия. E-mail: arefeva@mi-ras.ru, volovich@mi-ras.ru, inozemcev@mi-ras.ru 
Отметим, что в последние годы различные аспекты корреляций и динамики квантовых зацепленных состояний рассматривались в многочисленных работах (см., например, [2]-[6] и приведенные в них ссылки).

\section{2. ГОЛОГРАФИЧЕСКАЯ ЭНТРОПИЯ ЗАЦЕПЛЕННОСТИ В ПРОСТРАНСТВЕ ВАДЬИ-АДС}

Мы используем голографический подход для изучения эволюции открытой системы после квантового воздействия, сопровождающегося неравновесным процессом нагревания. В качестве дуальной модели, описывающей эволюцию энтропии зацепленности при таком нагреве, мы рассматриваем оболочку Вадьи, коллапсирующую на черную дыру [7], [8], [9]. Коллапс этой оболочки приводит к образованию более тяжелой черной дыры, что соответствует увеличению температуры. Начальное термическое состояние определяется положением горизонта $z_{\mathrm{H}}$, а конечное состояние положением горизонта $z_{\mathrm{h}}$. Для простоты мы рассматриваем трехмерный случай. Соответствующая метрика Вадьи, которая определяет дуальный гравитационный фон, состоит из двух частей и задается формулами

$$
\begin{array}{ll}
v<0: & d s^{2}=\frac{1}{z^{2}}\left[-\left(1-\frac{z^{2}}{z_{\mathrm{H}}^{2}}\right) d v^{2}-2 d v d z+d x^{2}\right], \\
v>0: & d s^{2}=\frac{1}{z^{2}}\left[-\left(1-\frac{z^{2}}{z_{\mathrm{h}}^{2}}\right) d v^{2}-2 d v d z+d x^{2}\right],
\end{array}
$$

где $0<z_{\mathrm{h}}<z_{\mathrm{H}}$, а склейка производится по положению оболочки $v=0$.

Чтобы определить голографическую энтропию зацепленности [10], [11], соответствующую простейшей системе из одного отрезка, нужно найти геодезическую в указанной метрике, закрепленную на этом отрезке в заданный момент времени. Действие для геодезической, соединяющей две точки на границе $(t,-\ell / 2)$ и $(t, \ell / 2)$ имеет вид (подробнее см. в [12], [13])

$$
S=\ln \left(\frac{z_{\mathrm{h}}}{\ell \mathfrak{S}_{\kappa}(\rho, s)} \operatorname{sh} \frac{\tau}{z_{\mathrm{h}}}\right), \quad 0 \leqslant t \leqslant \ell,
$$

где

$$
\mathfrak{S}_{\kappa}(\rho, s)=\frac{c \rho+\Delta}{\Delta} \sqrt{\frac{\Delta^{2}-c^{2} \rho^{2}}{\rho\left(c^{2} \rho+2 c \Delta+\rho\right)-\kappa^{2}}}
$$

и $\kappa=z_{\mathrm{h}} / z_{\mathrm{H}}<1, c=\sqrt{1-s^{2}}, \Delta=\sqrt{\rho^{2}-\kappa^{2}}$. Функция $\mathfrak{S}_{\kappa}(\rho, s)$ зависит от переменных $\rho, s$ (о физическом смысле этих переменных см. работы [12], [13]), которые связаны с переменными $t, \ell$ следующими явными выражениями:

$$
\begin{gathered}
\frac{t}{z_{\mathrm{h}}}=\operatorname{arcth} \frac{-c \kappa^{2}+2 c \rho^{2}+c+2 \Delta \rho}{2 c \rho+2 \Delta} \\
\ell=\frac{z_{\mathrm{h}}}{2} \ln \frac{c^{2} \gamma^{4}-4 \Delta\left(c s\left(\kappa^{2}-2 \rho^{2}+1\right)+\Delta+\Delta\left(\rho^{2}-2\right) s^{2}\right)}{c^{2} \gamma^{4}-4 \Delta^{2}(\rho s-1)^{2}}+\frac{z_{\mathrm{h}}}{2 \kappa} \ln \frac{(c \kappa+\Delta s)^{2}}{\rho^{2} s^{2}-\kappa^{2}},
\end{gathered}
$$

где

$$
\gamma=1-\kappa^{2}, \quad s=\frac{z_{c}}{z_{*}}, \quad \rho=\frac{z_{\mathrm{h}}}{z_{c}},
$$

причем удовлетворяются неравенства $z_{*}<z_{\mathrm{H}}$ и $z_{c}<z_{\mathrm{H}}$.

Нас интересует вычисление $S=S(\ell, t)$. Сначала найдем $\rho=\rho(\ell, t)$ и $s=s(\ell, t)$ из уравнений (4), а затем численно рассчитаем $S=S(s(\ell, t), \rho(\ell, t))=S(\ell, t)$. 


\section{3. ОСНОВАННЫЕ НА ЭНТРОПИИ ВЕЛИЧИНЫ ДЛЯ СИСТЕМ, СОСТОЯЩИХ ИЗ МНОГИХ ЧАСТЕЙ}

3.1. Взаимная информация. Напомним, что для произвольного двухчастичного квантового состояния $\rho_{A B}$ квантовая взаимная информация определяется как [2]

$$
I(A: B)=S\left(\rho_{A}\right)+S\left(\rho_{B}\right)-S\left(\rho_{A B}\right),
$$

где $S\left(\rho_{A}\right)=-\operatorname{Tr}\left(\rho_{A} \ln \rho_{A}\right)$ - энтропия зацепленности, $\rho_{A}=\operatorname{Tr}_{B}\left(\rho_{A B}\right)$ - редуцированная матрица плотности. На рис. 1 взаимная информация $I(A: B)$ соответствует $b+d$. Она показывает общую величину корреляции (как классической, так и квантовой) между подсистемами $A$ и $B$, измеряемой минимальной степенью случайности, которая требуется для полного устранения всех корреляций в $\rho_{A B}$ [14].

Если все слагаемые в правой части равенства (6) вычисляются голографически в соответствии с предыдущим разделом, то мы получаем голографическую взаимную информацию для системы, состоящей из двух частей:

$$
I(A: B)=S(A)+S(B)-S(A B)
$$

где $S(A)$ - (голографическая) энтропия Рю-Такаянаги отрезка $A$, через $A B$ обозначено $A \cup B$, а $S(A B)$ - голографическая энтропия зацепленности объединения двух отрезков. В работах [15], [16] было доказано, что взаимная информация всегда неотрицательна: $I(A: B) \geqslant 0$. Это свойство называется субаддитивностью энтропии.

3.2. Трехчастичная информация. Взаимная информация для системы, состоящей из трех частей (трехчастичная информация, информация взаимодействия), обычно определяется как

$$
\begin{aligned}
T I(A: B: C) & =I(A: B)+I(A: C)-I(A: B C)= \\
& =S(A)+S(B)+S(C)-[S(A B)+S(B C)+S(A C)]+S(A B C),
\end{aligned}
$$

где $A B C$ обозначает $A \cup B \cup C$. На рис. 1 трехчастичная информация равна $d$.

Как было показано в работе [17], для общей квантовой системы трехчастичная информация может быть положительной, или отрицательной, или равняться нулю в зависимости от выбора $A, B, C$. В работе [18] было доказано, что для любых отрезков $A, B, C$ голографическая трехчастичная информация неположительна: $T I(A: B: C) \leqslant 0$. Это неравенство может быть записано в форме

$$
I(A: B)+I(A: C) \leqslant I(A: B C) .
$$

Неравенства вида (9) часто возникают в квантовой теории информации и квантовой криптографии и связаны с отношениями "моногамности". Так, про голографическую трехчастичную информацию говорят, что она является моногамной [18]. Возникает вопрос, как это отрицательное значение TI может быть полезным для целей квантовой информации? Существует мнение [19], что отрицательность трехсторонней информации, которая представляет собой меру корреляции, является вызывающей. 


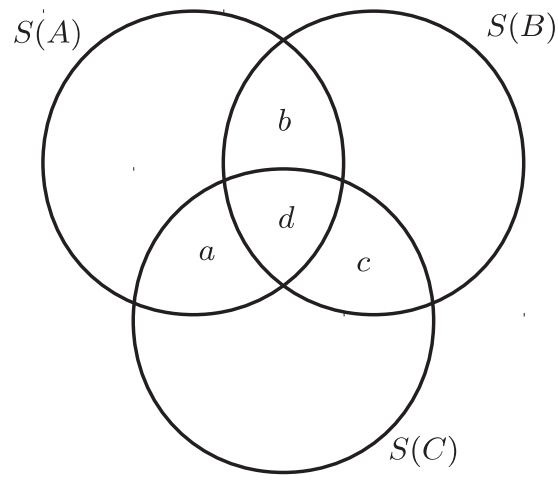

Рис. 1. Диаграмма Венна для системы, состоящей из трех частей; $I(A: B)$ соответствует $b+d, T I(A: B: C)$ соответствует $d, T C(A: B: C)$ соответствует $a+b+c+2 d, S M(A: B: C)$ соответствует $a+b+c+d$.

3.3. Полная корреляция. Полная корреляция представляет собой простое обобщение взаимной информации на многочастичный случай и определяется как

$$
T C\left(A_{1}: A_{2}: \cdots: A_{m}\right)=\sum_{i=1}^{m} S\left(A_{i}\right)-S\left(A_{1} A_{2} \ldots A_{m}\right) .
$$

На рис. 1 полная корреляция соответствует $a+b+c+2 d$. Эта величина использовалась, например, в [14], [20]-[23], а также в [24], где она называлась второй защищенной монотонностью.

Полная корреляция может быть представлена как сумма двухчастичных взаимных информаций [24]:

$$
T C\left(A_{1}: A_{2}: \cdots: A_{m}\right)=I\left(A_{1}: A_{2}\right)+\sum_{i=2}^{m-1} I\left(A_{1}: \cdots: A_{i}: A_{i+1}\right) .
$$

Так как взаимная информация неотрицательна, полная корреляция также неотрицательна: $T C\left(A_{1}: A_{2}: \cdots: A_{m}\right) \geqslant 0$.

3.4. Защищенная монотонность. Авторы работы [24], исследуя многочастичную квантовую криптографию, ввели величину, которую назвали защищенная монотонность (secrecy monotone). Такая же величина была независимо предложена и проанализирована Кумаром [19]. Кроме того, она использовалась в [22], [23], [25]. В литературе ее иногда называют дуальной полной корреляцией, или связующей информацией, или операционной квантовой взаимной информацией.

Для системы, состоящей их трех частей, защищенная монотонность задается как

$$
S M(A: B: C)=S(A B)+S(B C)+S(A C)-2 S(A B C) .
$$

На рис. 1 защищенная монотонность соответствует $a+b+c+d$.

Обобщение для системы, состоящей из $m$ частей, имеет вид

$$
S M\left(A_{1}: A_{2}: \cdots: A_{m}\right)=\sum_{i=1}^{m} S\left(A_{1} \ldots \check{A}_{i} \ldots A_{m}\right)-(m-1) S\left(A_{1} A_{2} \ldots A_{m}\right),
$$




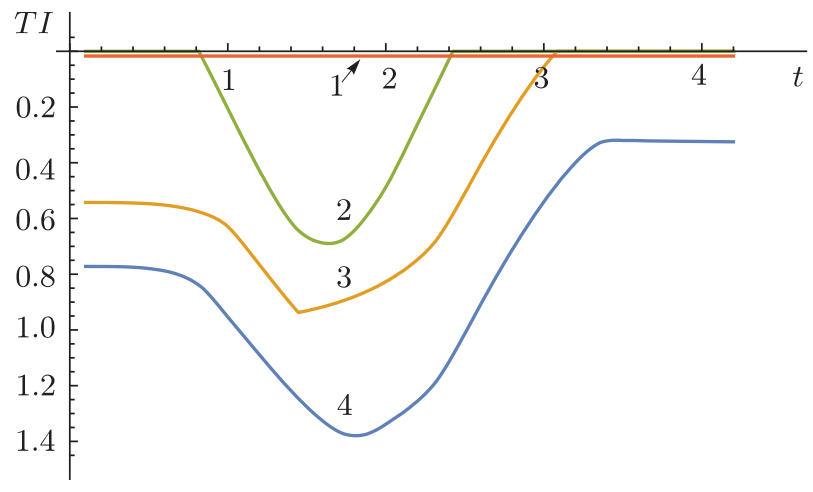

Рис. 2. Четыре типа поведения голографической трехчастичной информации.

где $\check{A}_{i}$ означает, что $A_{i}$ опускается. В статьях [19], [24] было показано, что защищенная монотонность неотрицательна: $S M\left(A_{1}: A_{2}: \cdots: A_{m}\right) \geqslant 0$. Заметим, что для системы, состоящей их двух частей, защищенная монотонность, очевидно, равна взаимной информации:

$$
S M(A: B)=S(A B)+S(B)+S(A)-2 S(A B)=I(A: B) .
$$

\section{4. ПОВЕДЕНИЕ ВЕЛИЧИН, ОСНОВАННЫХ НА ЭНТРОПИИ ЗАЦЕПЛЕННОСТИ}

В этом разделе мы исследуем зависимость указанных выше величин от времени для системы, состоящей из трех отрезков.

4.1. Трехчастичная информация. Мы обнаружили следующие четыре типа поведения голографической трехчастичной информации (рис. 2):

1) время пробуждения и время скремблинга отсутствуют, голографическая трехчастичная информация всегда отрицательна;

2) время пробуждения отсутствует, но есть время скремблинга;

3) есть время пробуждения и время скремблинга, график голографической трехчастичной информации имеет форму колокола;

4) голографическая трехчастичная информация тождественно равна нулю.

Конкретный вид графика голографической трехчастичной информации зависит от всех геометрических параметров (длин отрезков и расстояний между ними) системы и от температуры. В частности, при увеличении параметра $z_{\mathrm{H}}$, увеличении длин отрезков, уменьшении расстояний между ними абсолютное значение трехчастичной информации увеличивается.

4.2. Полная корреляция и защищенная монотонность. Мы обнаружили следующие пять типов поведения голографической полной корреляции (рис. 3а) и голографической защищенной монотонности (рис. 3б):

1) время пробуждения и время скремблинга отсутствуют, обе величины всегда положительны;

2) время пробуждения отсутствует, но есть время скремблинга; 

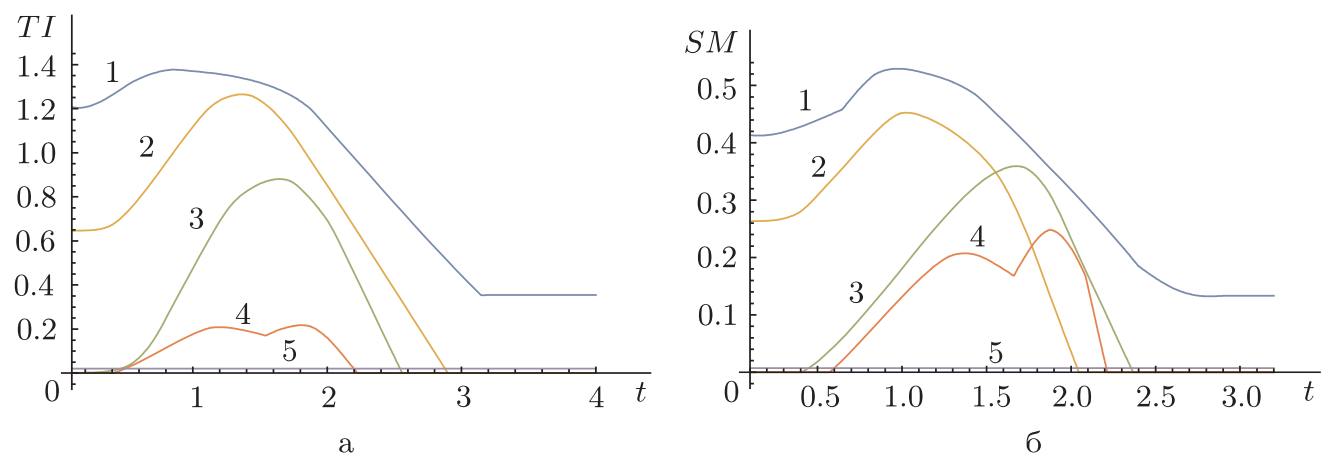

Рис. 3. Пять типов поведения голографической полной корреляции (а) и голографической защищенной монотонности (б).

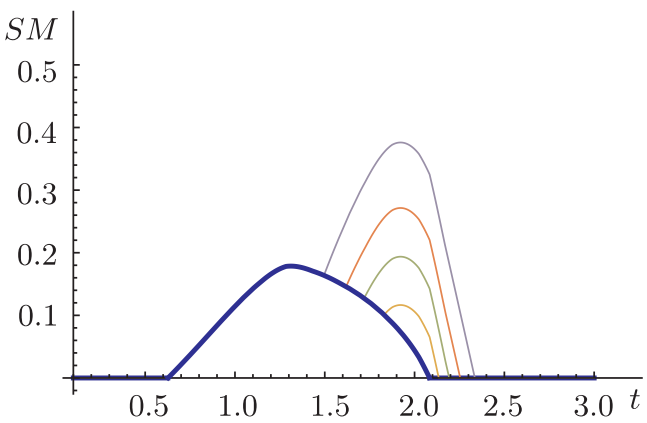

a

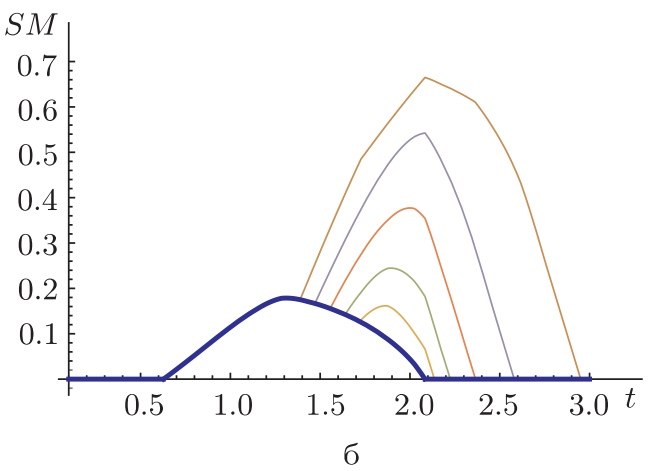

Рис. 4. Возникновение второго горба для графика голографической защищенной монотонности системы из трех отрезков.

3) есть время пробуждения и время скремблинга, графики обеих величин имеют форму колокола;

4) есть время пробуждения и время скремблинга, графики обеих величин имеют форму двойного горба;

5) обе величины тождественно равны нулю.

Конкретный вид графиков голографической полной корреляции и голографической защищенной монотонности зависит от всех геометрических параметров (длин отрезков и расстояний между ними) системы и от температуры. В частности, при увеличении параметра $z_{\mathrm{H}}$, увеличении длин отрезков, уменьшении расстояний между ними величи́ны полной корреляции и защищенной монотонности увеличиваются. Заметим, что указанные пять типов поведения в точности совпадают с поведением голографической взаимной информации для составной квантовой системы в [1]. Кроме того, похожие графики взаимной информации, полученные без использования голографического подхода, приведены в [26].

На рис. 4 показано возникновение формы двойного горба на графике голографической защищенной монотонности системы из трех отрезков. Пусть два отрезка $A$ 
и $B$ таковы, что график соответствующей им голографической защищенной монотонности имеет форму колокола (жирная линия). На рис. 4а показано появление второго горба по мере приближения отрезка $C$ к отрезкам $A$ и $B$ справа с достаточно большого расстояния. На рис. $4 б$ показано появление второго горба при увеличении длины отрезка $C$, находящегося на фиксированном расстоянии от отрезков $A$ и $B$.

\section{5. ЗАКЛЮЧЕНИЕ}

Используя голографический подход, мы изучили временную эволюцию величин трехчастичной информации, полной корреляции и защищенной монотонности для систем, состоящих из трех частей. Мы показали, как можно контролировать указанные величины, изменяя геометрическую конфигурацию системы и температуру. Были найдены четыре типа поведения временной зависимости голографической трехчастичной информации (рис. 2): трехчастичная информация отрицательна при всех временах; нет времени пробуждения, но есть время скремблинга; график имеет форму колокола; трехчастичная информация тождественно равна нулю.

Для временной зависимости голографической полной корреляции (рис. 3а) и голографической защищенной монотонности (рис. 3б) обнаружены следующие пять типов поведения: обе величины положительны при всех временах; нет времени пробуждения, но есть время скремблинга; графики обеих величин имеют форму колокола; графики обеих величин имеют форму двойного горба; обе величины тождественно равны нулю. Показано, что при изменении параметров системы можно получить колоколообразную и двугорбую формы графиков голографической полной корреляции и голографической защищенной монотонности. Колоколообразная форма и особенно двугорбая форма достигаются на довольно узких диапазонах параметров системы.

\section{Список литературы}

[1] И. Я. Арефьева, И. В. Волович, О.В.Иноземцев, "Голографический контроль информации и динамическое изменение топологии составных открытых квантовых систем", TMФ, 193:3 (2017), 493-504.

[2] M. Ohya, I. Volovich, Mathematical Foundations of Quantum Information and Computation and Its Applications to Nano- and Bio-systems, Springer, Dordrecht, 2011.

[3] A.S. Trushechkin, I. V. Volovich, "Perturbative treatment of inter-site couplings in the local description of open quantum networks", Europhys. Lett., 113:3 (2016), 30005, 6 pp.

[4] S. V. Kozyrev, A. A. Mironov, A. E. Teretenkov, I. V. Volovich, "Flows in non-equilibrium quantum systems and quantum photosynthesis", Infin. Dimens. Anal. Quantum Probab. Relat. Top., 20:4 (2017), 1750021, 19 pp., arXiv: 1612.00213.

[5] И. В. Волович, С. В. Козырев, "Манипуляция состояниями вырожденной квантовой системы", Тр. МИАН, 294 (2016), 256-267.

[6] I. V. Volovich, "Cauchy-Schwarz inequality-based criteria for the non-classicality of sub-Poisson and antibunched light", Phys. Lett. A, 380:1 (2016), 56-58.

[7] I. Aref'eva, I. Volovich, Holographic photosynthesis, arXiv: 1603.09107.

[8] И.Я. Арефьева, "Голографическое описание кварк-глюонной плазмы, образующейся при столкновениях тяжёлых ионов", УФН, 184:6 (2014), 569-598.

[9] I. Ya. Aref'eva, I. V. Volovich, "Holographic photosynthesis and entanglement entropy", International Conference on Infinite Dimensional Analysis, Quantum Probability and Related Topics, QP38 (Tokyo University of Science, Japan, 1-7 October, 2017), 2019 (to appear). 
[10] S. Ryu, T. Takayanagi, "Aspects of holographic entanglement entropy", JHEP, 08 (2006), 045, 47 pp., arXiv: hep-th/0605073.

[11] V.E. Hubeny, M. Rangamani, T. Takayanagi, "A covariant holographic entanglement entropy proposal", JHEP, 07 (2007), 062, 64 pp., arXiv: 0705.0016.

[12] Д. С. Агеев, И. Я. Арефьева, "Пробуждение и скремблинг в процессе голографического нагрева", ТМФ, 193:1 (2017), 146-161, arXiv: 1701.07280.

[13] D. S. Ageev, I. Y. Aref'eva, "Holographic non-equilibrium heating", JHEP, 03 (2018), 103, 18 pp., arXiv: 1704.07747.

[14] B. Groisman, S. Popescu, A. Winter, "Quantum, classical, and total amount of correlations in a quantum state", Phys. Rev. A, 72:3 (2005), 032317, 11 pp., arXiv: quant-ph/0410091.

[15] Д. Рюэль, Статистическая механика. Строгие результатьь, Мир, М., 171.

[16] H. Araki, E. H. Lieb, "Entropy inequalities", Commun. Math. Phys., 18:2 (1970), 160-170.

[17] H. Casini, M. Huerta, "Remarks on the entanglement entropy for disconnected regions", JHEP, 03 (2009), 048, 19 pp., arXiv: 0812.1773.

[18] P. Hayden, M. Headrick, A. Maloney, "Holographic mutual information is monogamous", Phys. Rev. D, 87:4 (2013), 046003, 11 pp., arXiv: 1107.2940.

[19] A. Kumar, "Multiparty quantum mutual information: an alternative definition", Phys. Rev. A, 96:1 (2017), 012332, 5 pp., arXiv: 1504.07176.

[20] S. Watanabe, "Information theoretical analysis of multivariate correlation", IBM J. Res. Develop., 4:1 (1960), 66-82.

[21] R. Horodecki, "Informationally coherent quantum systems", Phys. Lett. A, 187:2 (1994), $145-150$.

[22] D. Yang, K. Horodecki, M. Horodecki, P. Horodecki, J. Oppenheim, W. Song, "Squashed entanglement for multipartite states and entanglement measures based on the mixed convex roof", IEEE Trans. Inform. Theory, 55:7 (2009), 3375-3387, arXiv: 0704.2236.

[23] М. Е. Широков, "Меры корреляций в бесконечномерных квантовых системах", Матем. cб., 207:5 (2016), 93-142.

[24] N. J. Cerf, S. Massar, S. Schneider, "Multipartite classical and quantum secrecy monotones", Phys. Rev. A, 66:4 (2002), 042309, 13 pp., arXiv: quant-ph/0202103.

[25] S. Sazim, P. Agrawal, Quantum mutual information and quantumness vectors for multi-qubit systems, arXiv: 1607.05155 .

[26] K. Bradler, M. M. Wilde, S. Vinjanampathy, D. B. Uskov, "Identifying the quantum correlations in light-harvesting complexes", Phys. Rev. A, 82:6 (2010), 062310, 12 pp., arXiv: 0912.5112 . 